\title{
Corrélations entre méthode de préparation, hauteur de barrière et états d'interface dans les contacts métal-GaAs
}

\author{
C. Barret, F. Chekir, T. Neffati et A. Vapaille \\ Institut d'Electronique Fondamentale, Université Paris-XI, Bât. 220, 91405 Orsay Cedex, France
}

(Reçu le 25 juillet 1983, accepté le ler août 1983)

\begin{abstract}
Résumé. - La distribution des états d'interface dans un contact métal-semiconducteur peut être déterminée à partir de l'étude du comportement de la capacité dynamique d'une diode Schottky sous polarisation directe en fonction de la fréquence, la température et la tension appliquée. Cette méthode, appelée Spectroscopie de Capacité Schottky est appliquée à des dépôts d'Ag, $\mathrm{Au}$ et $\mathrm{Al}$ sur des surfaces de $\mathrm{GaAs}$ préparées de manières très diverses : clivage sous ultravide ou sous gaz, clivage suivi de bombardement ionique, nettoyage chimique, épitaxie par jets moléculaires. Ceci permet de tester le rôle joué par un grand nombre de paramètres sur la distribution des états d'interface et la hauteur de barrière. On constate que ces propriétés ne sont affectées de manière importante que par la création d'une zone interfaciale fortement perturbée, s'étendant sur plusieurs couches atomiques.
\end{abstract}

\begin{abstract}
Interface states distribution in metal-semiconductor contacts can be determined by studying variations of Schottky diode capacitance as a function of frequency, temperature and forward bias. This method, called Schottky Capacitance Spectroscopy is applied to contacts between $\mathrm{Ag}, \mathrm{Au}, \mathrm{Al}$ and diversely prepared surfaces : cleavage under ultra-high vacuum or controlled gazeous atmosphere, cleavage followed by ion-bombardment, chemical etch, molecular beam epitaxy. So, the rôle played by a great number of physical parameters on interface states distribution and barrier height can be determined. It is established that only the creation of a severely perturbed interfacial zone of a few angströms width can significantly modify the properties of the barrier.
\end{abstract}

\section{Introduction.}

Malgré les progrès effectués au cours des dernières années [1-4], la compréhension des mécanismes physiques conduisant à l'établissement d'une barrière de potentiel à l'interface entre un métal et un semiconducteur est encore imparfaite. Les états électroniques d'interface sont reconnus depuis longtemps [5] jouer un rôle majeur dans ces mécanismes. Mais les caractéristiques de ces états et, en premier lieu, leur distribution énergétique sont très mal connues.

Nous avons montré [6] qu'il est possible de faire varier la charge portée par ces états par modulation de la tension appliquée, à condition que la diode soit sous polarisation directe. Cette variation de charge interfaciale provoque l'apparition d'une composante capacitive dans l'admittance de la diode. L'étude du comportement de cette capacité avec la tension statique appliquée, la température et la fréquence du signal de test, permet d'obtenir des informations quantitatives sur la distribution énergétique des états et leur cinétique de charge et décharge. L'intérêt de cette méthode, appelée Spectroscopie de Capacité Schottky (SCS), est de pouvoir s'appliquer à une diode Schottky réelle à métallisation épaisse, alors que les méthodes optiques, telles que la spectroscopie de photoémission UV [2, 4], sont limitées à des surfaces préparées sous ultravide et recouvertes de moins d'une couche monoatomique de métal.

Nous avons étudié par SCS des interfaces métalsemiconducteur réalisées par dépôt d'aluminium, d'or ou d'argent, sur des surfaces de GaAs obtenues de façons très diverses : clivage sous ultravide ou sous oxygène; clivage suivi d'un bombardement ionique in situ; nettoyage chimique; épitaxie par jet moléculaire. Ceci permet de dégager d'éventuelles corrélations entre la distribution des états d'interface, la hauteur de barrière et les paramètres physiques affectés par la méthode de préparation.

\section{La méthode SCS.}

2.1 PRINCIPE. - Supposons qu'un état d'interface discret soit situé entre le niveau de Fermi du métal $E_{F_{M}}$ et le bas de la bande de conduction $E_{\mathrm{c}}$ d'un semiconducteur de type $n$ (cf. Fig. 1). La charge $Q_{\text {is }}$ portée par cet état résulte du bilan des échanges d'électrons et de trous entre l'état d'une part et, d'autre part : la bande de conduction du semiconducteur, sa bande de valence et la bande de conduction du métal. En 


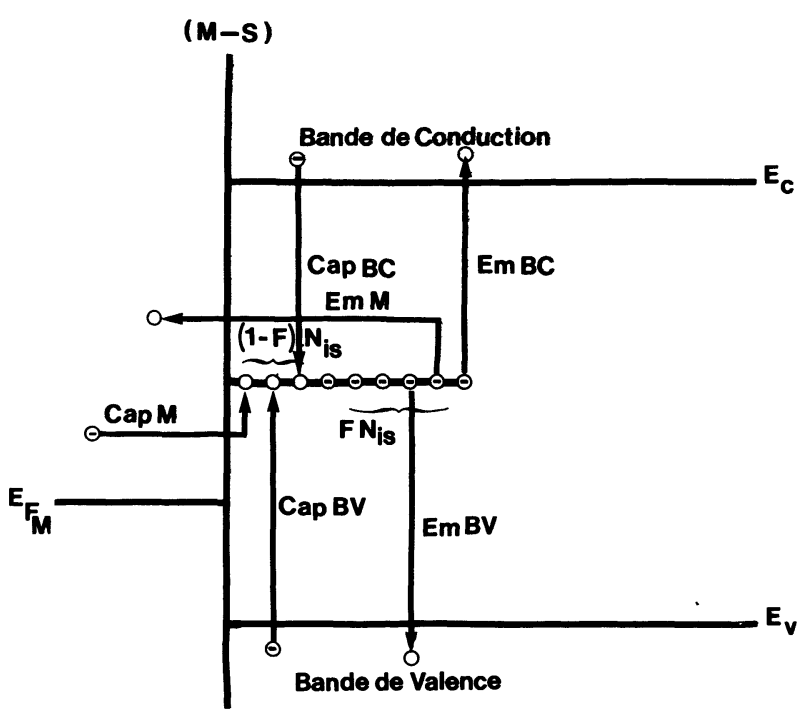

Fig. 1. - Schéma des différents mécanismes d'échange de charge entre un état d'interface et les réservoirs de charge qui l'entourent. (Cap : capture, Em : émission; BC : bande de conduction; $\mathrm{BV}$ : bande de valence.)

[Diagram of the various types of charge-exchange mechanisms between an interface state and the charge reservoirs surrounding it. (Cap : capture ; Em : emission ; BC : conduction band; BV : valence band).]

régime permanent continu, un état d'équilibre s'établit et la fraction $F$ des états occupée par un électron est constante. Mais cette fraction $F$ est sensible à la tension de polarisation directe $V_{0}$ appliquée à la diode. En effet, on sait que pour un contact entre un métal et un semiconducteur covalent tel que $\mathrm{Si}$, GaAs ou InP, la conduction des électrons s'effectue par émission thermoionique au-dessus de la barrière de potentiel à l'interface. Une conséquence directe de ce fait est que [7] le quasi-niveau de Fermi des électrons se déplace à l'interface de telle sorte que

$$
E_{\mathrm{F}_{\mathrm{ns}}}-E_{\mathrm{F}_{\mathrm{M}}}=q V_{0} \text { ou } E_{\mathrm{c}}-E_{\mathrm{F}_{\mathrm{ns}}}=\phi_{\mathrm{B}_{\mathrm{n}}}-q V_{0}
$$

( $\phi_{\mathrm{B}_{\mathrm{n}}}$ étant la hauteur de la barrière de Schottky).

Ceci revient à dire que la concentration $n_{\mathrm{s}}$ des électrons présents dans la bande de conduction au voisinage de l'interface varie exponentiellement avec la tension appliquée. Or, la probabilité qu'un de ces électrons soit capturé par l'état d'interface dépend naturellement de $n_{s}$. En modulant $V_{0}$ par un petit signal sinusoïdal, on module $n_{s}$, donc la fonction d'occupation $F$ des états et par suite la charge interfaciale. La conséquence sur les caractéristiques du dispositif est qu'une capacité excédentaire $C_{\text {is }}$ s'ajoute à la capacité $C_{\mathrm{SC}}$ due à la zone de charge d'espace. La figure 2 montre la variation de la capacité totale d'une jonction Ag-GaAs clivé en fonction de la fréquence à diverses polarisations. A fréquence élevée, la capacité est constante et égale à la capacité de zone de charge d'espace. Lorsque la fréquence diminue, on voit se superposer à $C_{\mathrm{SC}}$ la capacité $C_{\mathrm{is}}$ due aux

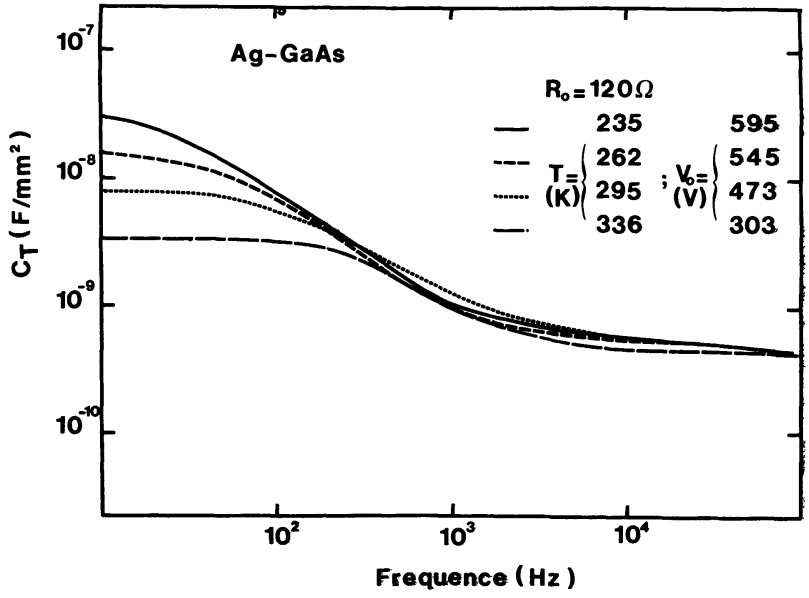

Fig. 2. - Variations de la capacité totale d'une jonction Ag-GaAs $<110>$ clivé en fonction de la fréquence à diverses polarisations.

[Capacitance variations of an Ag-GaAs (UHV cleaved) junction as a function of frequency at various forward biases.]

états d'interface. Cette capacité croît lorsque la fréquence diminue et se sature, en deçà d'une fréquence de coupure $f_{\mathrm{c}}$, à une valeur $C_{\mathrm{iso}}$ fonction de la polarisation $V_{0}$. Cela signifie que la charge des états d'interface ne peut suivre la modulation de la tension appliquée que si la période du signal de test est grande par rapport à leur temps de relaxation.

2.2 DisPositif EXPÉRIMENTAL. - La principale difficulté est qu'il faut mesurer une capacité à fréquence basse (inférieure à la fréquence de coupure) en présence d'une conductance élevée, puisque la jonction est en polarisation directe. La solution adoptée [6] consiste à inclure la diode dans un pónt constitué par trois résistances égales $R_{0}$ (cf. Fig. 3). Le pont est alimenté par une source de courant continu $I_{0}$ à laquelle est superposé un petit signal sinusoïdal. Pour éviter toute distorsion, l'amplitude de lá composante dynamique $v_{\mathrm{AC}}$ de la tension d'alimentation

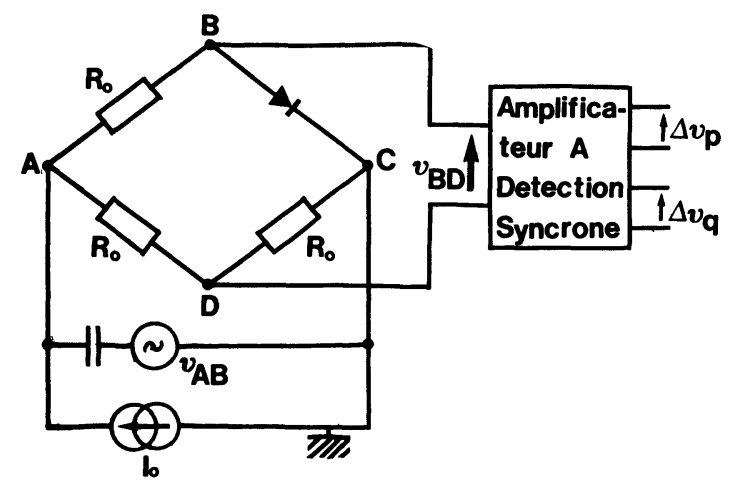

Fig. 3. - Schéma de principe du dispositif de mesure ae capacité.

[Scheme of the experimental set-up for capacitance measurements.] 
du pont est limitée à $2 \mathrm{mV}$. La tension d'erreur du pont $v_{\mathrm{BD}}$ est analysée par un amplificateur à détection synchrone double phase qui sépare les composantes $\Delta V_{\mathrm{p}}$ et $\Delta V_{\mathrm{q}}$ en phase et en quadrature avec $v_{\mathrm{AC}}$. La source de courant $I_{0}$ est asservie de façon à maintenir $\Delta V_{\mathrm{p}}=0$. Un calcul élémentaire d'analyse de réseau montre que cela revient à réaliser :

$$
G_{\mathrm{T}}=R_{0}^{-1}
$$

$\mathrm{G}_{\mathrm{T}}$ étant la conductance dynamique totale de la diode.

Le pont étant ainsi équilibré, la composante en quadrature résiduelle $\Delta V_{\mathrm{q}}$ est telle que :

$$
\Delta V_{\mathrm{q}}=V_{\mathrm{AC}} \cdot \frac{R_{0} C_{\mathrm{T}} \omega}{4},
$$

ce qui permet de déterminer la capacité totale $C_{\mathrm{T}}$ de la diode. Il est à noter que les relations 1 et 2 sont valides dans l'approximation $R_{0} C_{\mathrm{T}} \omega \ll 1$ qui est remplie très aisément dans les conditions expérimentales utilisées.

Un balayage de fréquence permet de relever une courbe $C_{\mathrm{T}}(f)$ du type de celles représentées sur la figure 2. De chaque courbe, on peut déduire la fréquence de coupure $f_{\mathrm{c}}$ et la limite basse fréquence $C_{\mathrm{is}}$ de la capacité due à la réponse des états d'interface. Ces valeurs correspondent à une polarisation $V_{0}$ donnée, c'est-à-dire à une position donnée du quasiniveau de Fermi des majoritaires à l'interface. Pour se déplacer dans la bande interdite, il suffit de modifier la polarisation continue. Ceci peut se faire de deux façons :

-à température constante : il faut diminuer la valeur de $R_{0}$ au fur et à mesure que $V_{0}$ augmente;

- à conductance constante : on conserve fixes les résistances du pont et on refroidit la diode au fur et à mesure que $V_{0}$ augmente.

C'est généralement cette seconde solution qui est utilisée car, outre sa simplicité de mise en ouvre, elle présente l'avantage de fournir directement les variations de la fréquence de coupure avec la température. Nous verrons par la suite qu'une telle courbe donne des indications importantes pour l'analyse des résultats expérimentaux.

\subsection{ANAL ySE DES RÉSUltats. - Un exemple de résul-} tat expérimental pour une interface $\mathrm{Ag}-\mathrm{GaAs}$ clivé est donné sur les figures 4 et 5 . La figure 4 représente les variations avec la polarisation continue de la densité apparente des états d'interface $C_{\text {iso }} / q S, S$ étant l'aire de la jonction. La figure 5 représente les variations de la fréquence de coupure avec la température.

Le problème est maintenant de déterminer à partir de ces résultats les principales caractéristiques des états d'interface : densité, position énergétique, section efficace de capture. Une modélisation complète a été effectuée [8], dont nous ne rappellerons ici que les conclusions essentielles.

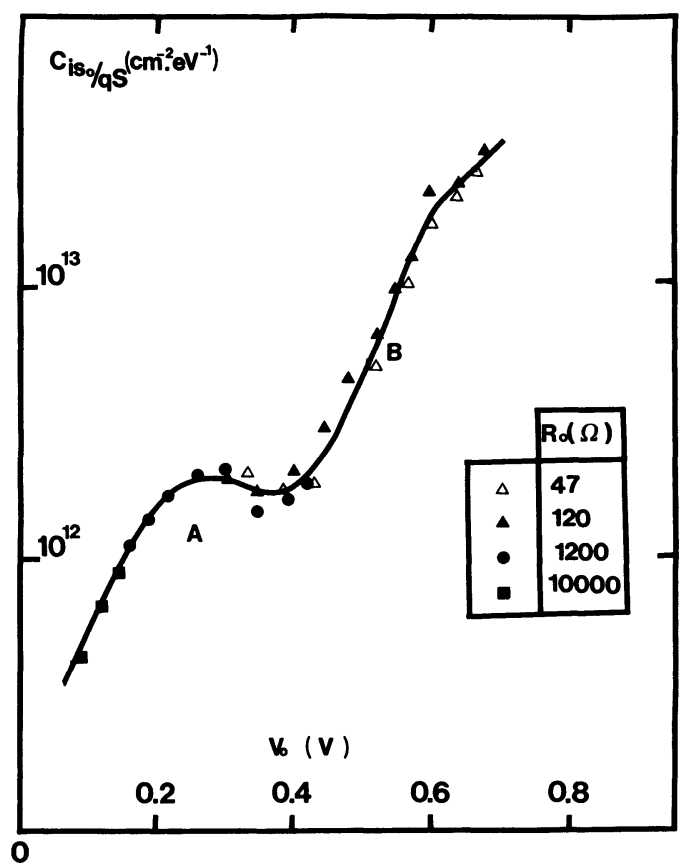

Fig. 4. - Variations de la densité apparente des états d'interface avec la polarisation continue pour une jonction $\mathrm{Ag}$ $\mathrm{GaAs}\langle 110\rangle$ clivé.

[Variations of the apparent density of interface states as a function of bias for an Ag-GaAs (UHV cleaved) junction.]

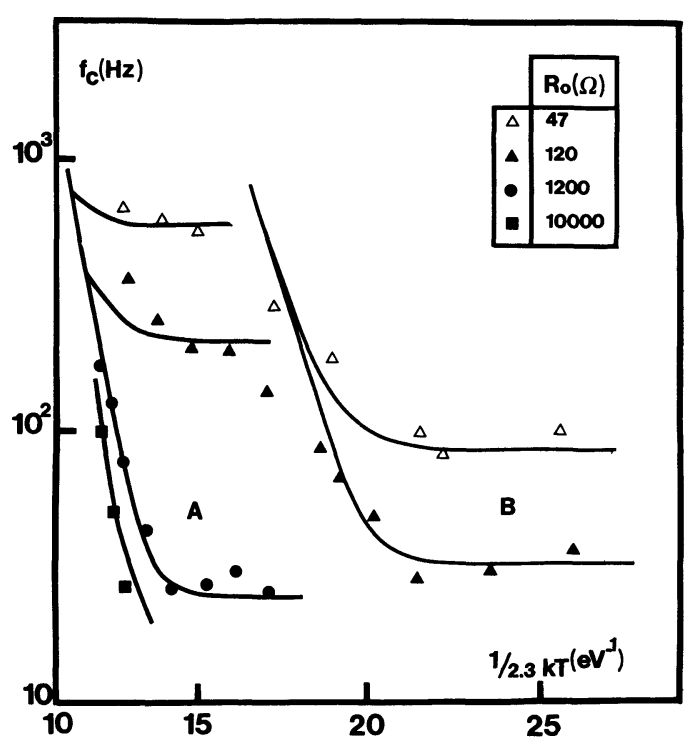

Fig. 5. - Variations de la fréquence de coupure avec la température pour la jonction Ag-GaAs $\langle 110\rangle$ clivé.

[Cut-off frequency variations as a function of temperature for the same junction.]

La courbe expérimentale $\frac{C_{\text {iso }}}{q S}\left(\phi_{\mathrm{B}}-V_{0}\right)$ est une image de la distribution énergétique réelle des états d'interface qui peut être plus ou moins déformée suivant le mécanisme le plus efficace pour la décharge de l'état. 
Sur la figure 6 est simulée la variation de $C_{\text {iso }} / q S$ correspondant à une bande d'états de densité constante $N_{\mathrm{is}}$ entre deux limites énergétiques $E_{\mathrm{s}_{1}}$ et $E_{\mathrm{s}_{2}}$.

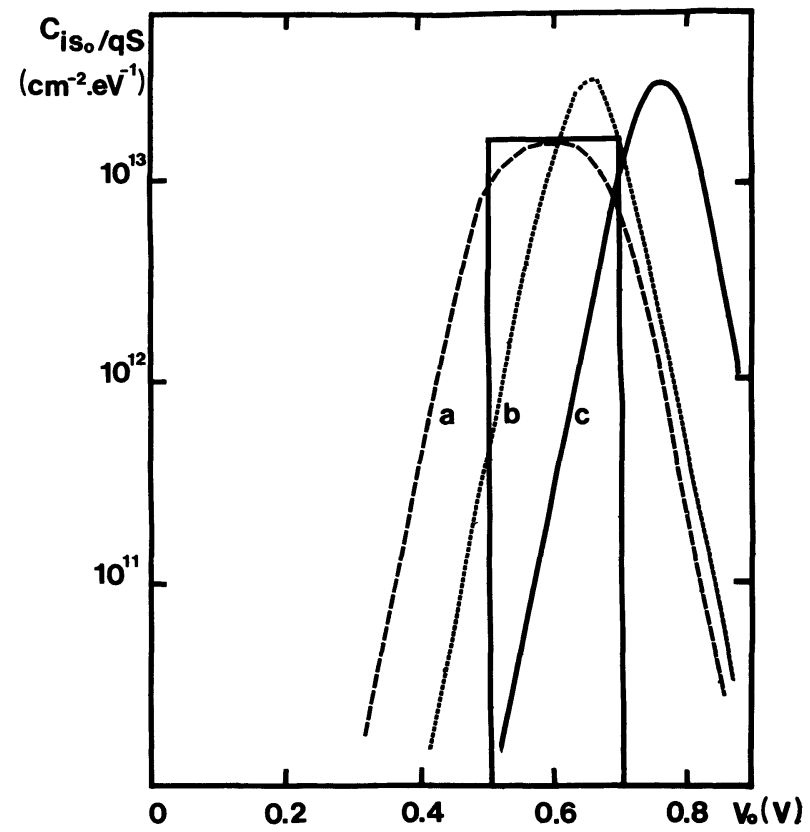

Fig. 6. - Variations de la capacité des états d'interface avec la polarisation dans le cas d'échanges de charge avec les deux bandes du semiconducteur. Simulations numériques avec : $\phi_{\mathbf{B}_{\mathbf{n}}}=0,95 \mathrm{eV} ; E_{\mathbf{s}_{1}}=0,45 \mathrm{eV} ; E_{\mathrm{s}_{2}}=0,25 \mathrm{eV}$; $N_{\text {is }}=6 \times 10^{12} \mathrm{~cm}^{-2} \mathrm{eV}^{-1} ; \frac{\sigma_{\mathrm{n}}}{\sigma_{\mathrm{p}}}=10^{3}$ (a), 1 (b), $10^{-3}$ (c).

[Numerical simulation of interface states capacitance variations as a function of bias in the case of charge-exchanges with both semiconductor bands.]

Les différentes courbes correspondent à des valeurs croissantes du rapport des sections efficaces de capture des électrons et des trous. Lorsque la probabilité de capture d'un trou est faible (courbe a), les variations de capacité donnent une image correcte de la distribution réelle des états à la présence près de flancs exponentiels de pente $k T$. Lorsque la capture des trous devient prépondérante (courbes $b, c$ ), la courbe expérimentale apparaît décalée vers la bande de conduction par rapport au spectre réel. La distinction entre les cas a d'une part, et $(b, c)$ d'autre part, peut s'effectuer en déterminant l'énergie d'activation de la fréquence de coupure (pente de $f_{\mathrm{c}} T^{-2}$ en fonction de $\left.q / k T\right)$ : elle vaut $E_{\mathrm{s}_{1}}$ dans le cas a, et $E_{\mathbf{G}}-\phi_{\mathbf{B}_{\mathbf{n}}}$ dans les cas b et c.

La figure 7 montre les variations de $C_{\text {iso }} / q S$ pour des probabilités croissantes d'effet tunnel entre les états et le métal. La courbe a correspond au cas où les électrons capturés par les états d'interface sont réémis préférentiellement vers la bande de conduction $\mathrm{du}$ semiconducteur. Cette courbe est identique à la courbe a de la figure 6 et est une image fidèle de la distribution réelle des états. Les courbes b et c correspondent au contraire à une réémission préférentielle

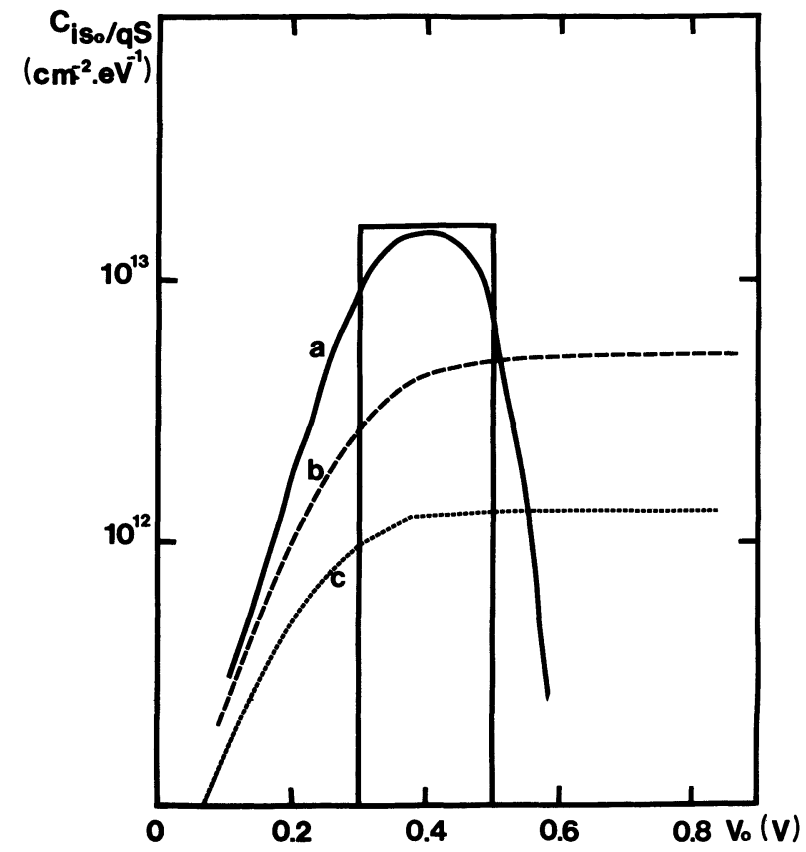

Fig. 7. - Cas d'échanges de charge avec les bandes de conduction du semiconducteur et du métal. Simulations numériques avec : $\phi_{\mathbf{B}_{\mathbf{n}}}=0,95 \mathrm{eV} ; \quad E_{\mathrm{s}_{1}}=0,65 \mathrm{eV}$; $E_{\mathrm{s}_{2}}=0,45 \mathrm{eV} ; N_{\mathrm{is}}=6.10^{12} \mathrm{~cm}^{-2} \mathrm{eV}^{-1} ; \sigma_{\mathrm{n}}=3.10^{-14} \mathrm{~cm}^{-2}$; $\sigma_{\mathrm{p}}=0$. Courbe a : $\tau_{\mathrm{T}}=10^{-2} \mathrm{~s} ; R_{0}=50 \Omega$ ou $100 \Omega$. Courbe b : $\tau_{\mathrm{T}}=2 \times 10^{-6} \mathrm{~s}$ et $R_{0}=100 \Omega$. Courbe c : $\tau_{\mathrm{T}}=2 \times 10^{-6}$ s et $R_{0}=400 \Omega$.

[Numerical simulation in the case of charge-exchanges with the semiconductor conduction band and the metal.]

vers le métal. On constate l'importante déformation par rapport à la distribution réelle $: C_{\text {iso }} / q S$ est très inférieur à la densité effective $N_{\text {is }}$ et la bande réelle apparaît étirée du côté de la bande de conduction du semiconducteur. On peut distinguer les cas a d'une part, $(b, c)$ d'autre part par le fait que, dans une expérience réalisée à conductance constante, la courbe a est insensible à la valeur de la résistance $R_{0}$ du pont de mesure, alors que le plateau des courbes $b$ et $\mathrm{c}$ lui est inversement proportionnel.

\section{Résultats expérimentaux.}

3.1 Préparation des ÉChantillons. - Nous utilisons de l'arséniure de gallium de type $\mathrm{n}$ dont le dopage est de l'ordre de $10^{16} \mathrm{~cm}^{-3}$ et dont la surface peut être préparée de multiples façons :

- clivage sous ultravide;

- clivage sous atmosphère contrôlée d'oxygène;

- clivage suivi d'un bombardement ionique léger

$\left(\mathrm{Ar}^{+}, 500 \mathrm{eV}\right)$

- nettoyage chimique.

Les métaux déposés sur ces surfaces sont l'or, l'argent ou l'aluminium. Les dépôts peuvent être effectués soit par évaporation flash, soit à vitesse très lente $(\leqslant 0,1$ monocouche par seconde). Les diodes réalisées peuvent éventuellement subir un 
Tableau I. - Principales caractéristiques électriques des différentes interfaces étudiées.

\begin{tabular}{|c|c|c|c|c|c|c|c|c|c|}
\hline \multirow{2}{*}{$\begin{array}{c}\text { Métal déposé } \\
\begin{array}{c}\text { Orientation } \\
\text { cristalline } \\
\text { du GaAs }\end{array}\end{array}$} & \multicolumn{4}{|c|}{$\mathrm{Ag}$} & \multicolumn{3}{|c|}{$\mathrm{Au}$} & \multicolumn{2}{|c|}{ Al } \\
\hline & \multicolumn{3}{|c|}{$\langle 110\rangle$} & \multirow{2}{*}{$\begin{array}{c}\langle 100\rangle \\
\begin{array}{c}\text { nettoyage } \\
\text { chimique }\end{array}\end{array}$} & \multicolumn{3}{|c|}{$\langle 110\rangle$} & \multirow{2}{*}{$\begin{array}{c}\langle 110\rangle \\
\begin{array}{c}\text { clivé sous } \\
\text { ultravide }\end{array}\end{array}$} & \multirow{2}{*}{$\begin{array}{r}\langle 100\rangle \\
\text { EJM }\end{array}$} \\
\hline Préparation & $\begin{array}{l}\text { clivé sous } \\
\text { ultravide }\end{array}$ & $\begin{array}{c}\text { clivé } \\
\text { sous } \mathrm{O}_{2}\end{array}$ & $\begin{array}{c}\text { clivé et } \\
\text { bombardé }\end{array}$ & & $\begin{array}{l}\text { clivé sous } \\
\text { ultravide }\end{array}$ & $\begin{array}{c}\text { clivé } \\
\text { sous } \mathrm{O}_{2}\end{array}$ & $\begin{array}{c}\text { recuit }\left(^{*}\right) \\
200^{\circ} \mathrm{C}-150 \mathrm{~min} .\end{array}$ & & \\
\hline$\phi_{\mathrm{BC}}(\mathrm{eV})$ & 0,95 & 0,91 & 0,61 & 0,91 & 0,95 & 0,9 & 0,95 & 0,89 & 0,82 \\
\hline$\phi_{\mathrm{BJ}}(\mathrm{eV})$ & 0,95 & 0,86 & 0,53 & 0,84 & 0,88 & 0,71 & 0,74 & 0,84 & 0,75 \\
\hline$n$ & 1,03 & 1,11 & 1,30 & 1,08 & 1,08 & 1,4 & 1,23 & 1,12 & 1,1 \\
\hline
\end{tabular}

${ }^{*}$ ) Traitement thermique réalisé sur une interface Au-GaAs clivée sous ultravide.

traitement thermique jusqu'à $300^{\circ} \mathrm{C}$ en atmosphère gazeuse contrôlée $\left(\mathrm{O}_{2}, \mathrm{H}_{2}, \ldots\right)$.

Un autre type d'échantillon a été obtenu par épitaxie par jet moléculaire (EJM) suivant la procédure suivante :

i) croissance épitaxiale sur un substrat fortement dopé d'une couche de GaAs (001) de quelques microns d'épaisseur;

ii) contrôle de la composition atomique superficielle de la couche par modification des conditions expérimentales (température, pression d'arsenic) en fin de croissance;

iii) dépôt progressif in situ de $3000 \AA$ d'aluminium.

3.2 HAUTEURS DE BARRIÈRE. - Elles sont déterminées de façon classique à partir des caractéristiques courant-tension et capacité-tension inverse. Le tableau I donne-les résultats obtenus à température ambiante. $\phi_{\mathrm{BJ}}$ est la hauteur de barrière déduite de la caractéristique $I-V ; n$ est le facteur de nonidéalité et $\phi_{\mathrm{BC}}$ est la hauteur de barrière déduite de la courbe $C^{-2}-V$.

3.3 ETATS D'INTERFACE. - Quelques spectres expérimentaux caractéristiques donnant les variations de la densité apparente d'états $C_{\text {is }} / q S$ en fonction de la polarisation (ou de la position du quasi-niveau de Fermi des électrons à l'interface) sont représentés sur les figures 8 à 10 .

- La première remarque concernant ces courbes est qu'elles font apparaître généralement l'existence de trois types d'états d'interface qui sont repérés sur les figures 8, 9 et 10, par les lettres A, B et C. Les caractéristiques générales de ces états sont les suivantes :

- Etats $A$ : ils sont systématiquement présents sur toutes les interfaces étudiées (sauf lorsque la surface a subi un bombardement ionique). Leur densité varie entre $10^{11} \mathrm{~cm}^{-2} \cdot \mathrm{eV}^{-1}$ et $2 \times 10^{12} \mathrm{~cm}^{-2} \cdot \mathrm{eV}^{-1}$. L'étude des variations des fréquences de coupure avec la température, montre que ces états ont une probabilité importante de se décharger par capture de trous. En conséquence, les spectres expérimentaux sont du type des courbes de simulation b ou c de la figure 6. La position énergétique réelle de ces états ne peut être déterminée avec exactitude. Ils se trouvent en dessous de $E_{\mathrm{c}}-0,65 \mathrm{eV}$ et il est possible qu'ils s'étendent jusqu'au niveau de Fermi du métal et même au-delà. Ils jouent donc probablement un rôle important dans la fixation de la hauteur de barrière. On constate effectivement une corrélation entre la valeur de $\phi_{\mathbf{B}_{\mathbf{n}}}$ et la densité des états A : plus la densité est élevée, plus la barrière est élevée.

- Etats $B$ : ils sont situés autour de $E_{\mathrm{c}}-0,4 \mathrm{eV}$ et leur spectre expérimental apparaît soit sous forme d'un pic bien défini (Al-GaAs (001) C $8 \times 2)$, soit sous forme d'une zone à croissance monotone. Il n'est pas actuellement clairement établi si le changement de forme du spectre expérimental d'une interface à l'autre est dû à une modification effective de la distribution réelle des états, ou bien s'il s'agit d'une distorsion apparente plus ou moins accentuée due à des probabilités différentes d'effet tunnel avec le métal.

- Etats $C$ : ils se manifestent sur les courbes expérimentales par une zone à croissance exponentielle de pente voisine de $k T$. Cela signifie que les états réels ne sont pas encore atteints par le quasiniveau de Fermi des électrons et qu'ils sont situés au-delà de $E_{\mathrm{c}}-0,3 \mathrm{eV}$. On observe, à la différence des états A et $B$, une extrême sensibilité de cette partie du spectre expérimental aux conditions de réalisation des interfaces : sur la figure 8 , on constate que ces états existent avec une densité très élevée à l'interface Al-GaAs clivé, sont présents en faible densité à l'interface $\mathrm{Au}-\mathrm{GaAs}$ clivé et n'apparaissent pas pour l'interface Ag-GaAs clivé. De même, la figure 9 montre que leur densité diminue beaucoup si on prépare une jonction Al-GaAs par EJM, et non par clivage. Si les phénomènes influençant la densité des états $\mathrm{C}$ semblent être multiples, il apparaît, par contre, une claire corrélation entre la présence en densité élevée de ce type d'états et une dégradation de la caractéristique $I-V$ de la jonction. Ceci peut s'expliquer simplement si ces états, situés près du bas de la bande 


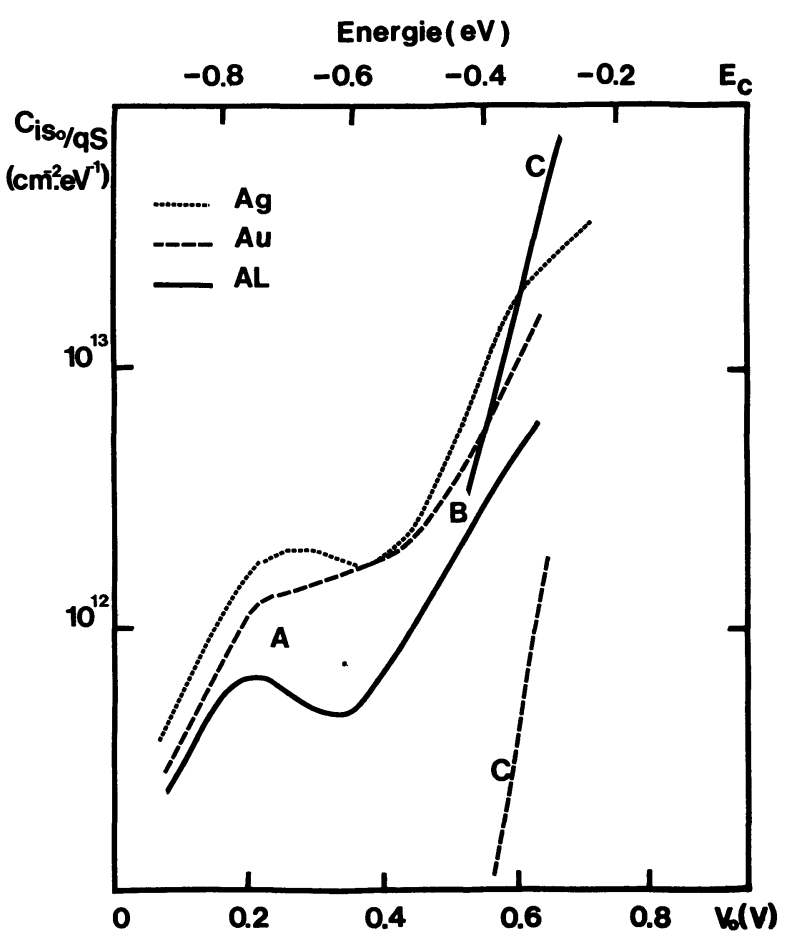

Fig. 8. - Interfaces $\mathrm{Au}, \mathrm{Ag}, \mathrm{Al}-\mathrm{GaAs}\langle 110\rangle$ clivé sous ultravide : variations de la densité apparente d'états d'interface en fonction de la polarisation (ou de la position énergétique du quasi-niveau de Fermi).

[Au, Ag, Al UHV cleaved GaAs $\langle 110\rangle$ interfaces : variations of the apparent density of interface states as a function of bias (or electron quasi-Fermi level position).]

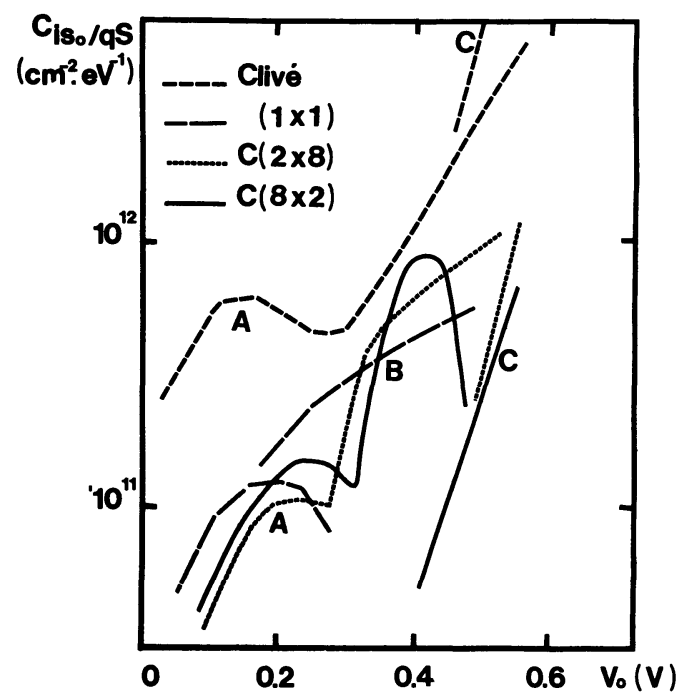

Fig. 9. - Variations de $C_{\text {iso }} / q S$ en fonction de la polarisation pour des dépôts d'Al sur des surfaces de GaAs clivées ou préparées par EJM (avec différentes surstructures).

[Variations of $C_{\text {iso }} / q S$ as a function of bias for interfaces between $\mathrm{Al}$ and GaAs surfaces obtained by cleavage or molecular beam epitaxy (with various surstructures).] de conduction du semiconducteur, servent de relais pour le passage des électrons par effet tunnel entre le semiconducteur et le métal.

- La seconde remarque est que pour modifier de façon très significative le spectre des états d'interface métal-GaAs, il faut créer, entre le métal et le semiconducteur une zone interfaciale fortement perturbée de plusieurs couches atomiques d'épaisseur. C'est le cas de la surface ayant subi un bombardement d'ions $\mathrm{Ar}^{+}$(cf. Fig. 10) pour laquelle n'apparaît plus qu'une large distribution d'états d'interface s'étendant sur toute la partie de la bande interdite explorable. Simultanément à cette profonde altération de la distribution des états d'interface, la barrière de Schottky est notablement diminuée (passant de $0,94 \mathrm{eV}$ à environ $0,6 \mathrm{eV}$ pour Ag-GaAs). Un comportement similaire est obtenu pour les contacts Au-GaAs clivé à la suite d'un traitement thermique de quelques minutes vers $200^{\circ} \mathrm{C}$ en atmosphère d'oxygène. Dans ce cas, la couche interfaciale épaisse et fortement perturbée, est due à l'interdiffusion de l'or (et éventuellement de l'oxygène) avec le GaAs.

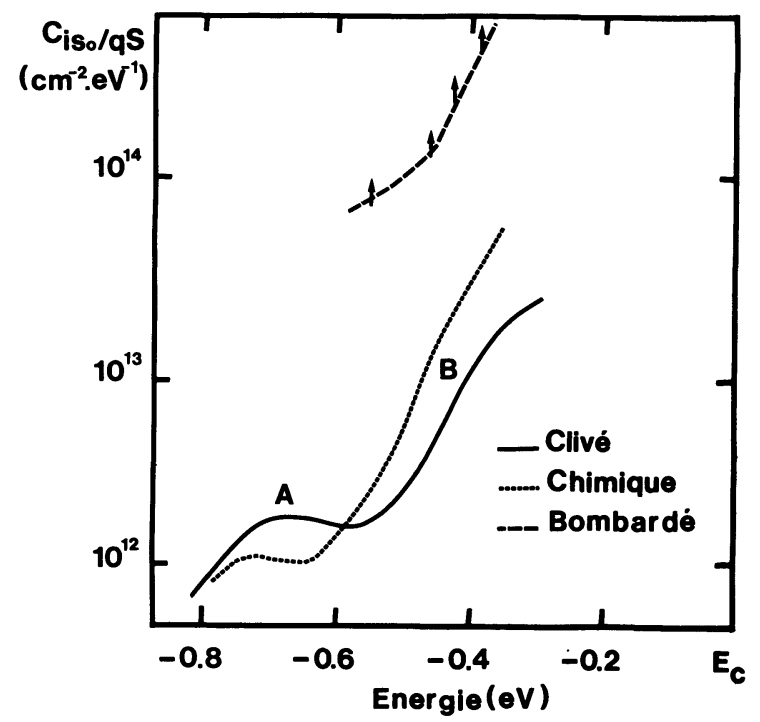

Fig. 10. - Variations de $C_{\text {is }} / q S$ en fonction de l'énergie pour des dépôts d'argent sur des surfaces de GaAs clivées, nettoyées chimiquement ou clivées et bombardées.

[Variations of $C_{\text {iso }} / q S$ as a function of energy for interfaces between $\mathrm{Ag}$ and $\mathrm{GaAs}$ surfaces obtained by cleavage, chemical etching or cleavage followed by ion-bombardment.]

\section{Discussion.}

Il est possible de déterminer l'influence de divers paramètres physiques sur la hauteur de barrière et la distribution des états d'interface.

4.1 Travall DE SORTIE DU métal. - Son rôle est négligeable. Le dépôt d'or $\left(\phi_{M}=5,2 \mathrm{eV}\right)$ ou d'argent $\left(\phi_{M}=4,3 \mathrm{eV}\right)$ sur la surface clivée du GaAs donne des barrières pratiquement identiques. De même, 
lors de la préparation des contacts Al-GaAs par EJM, deux orientations différentes, (001) et (011), ont pu être obtenues pour le film d'aluminium [9]. A ces deux orientations correspond un écart des travaux de sortie de $0,35 \mathrm{eV}$ [10], qui n'a aucune influence sur la barrière de Schottky.

4.2 Composition SUPERFICIELle DU GaAs. - La technique d'épitaxie par jet moléculaire a permis de réaliser des couches de GaAs à composition atomique superficielle variable et contrôlée [11]. La hauteur de barrière des diodes obtenues après dépôt d'aluminium sur ces couches ne montre pas de corrélation significative avec leur composition [12]. Les distributions d'états d'interface ont été relevées sur des contacts correspondant à des surfaces déplétées en arsenic (reconstruction C $8 \times 2$ ), quasi stœechiométriques (C $2 \times 8)$ et enrichies en arsenic $(1 \times 1)$. L'écart entre les trois spectres est faible (cf. Fig. 9) et porte essentiellement sur la forme de la zone $B$.

4.3 RÉACTIVITÉ DU MÉTAL. - Les spectres de la figure 8 montrent que la densité des états $\mathrm{A}$ et $\mathrm{B}$ est plus faible pour un métal réactif $(\mathrm{Al})$ que pour un métal non réactif $(\mathrm{Ag}, \mathrm{Au})$. A cette différence de densité d'états est associée une barrière de Schottky plus faible pour l'aluminium.

4.4 Croissance du métal. - Des dépôts d'argent sur $\mathrm{GaAs}$ clivé ont été réalisés soit à vitesse très lente ( $\leqslant 0,1$ monocouche/s) dans des conditions permettant une croissance ordonnée du métal, soit par évaporation flash. Aucune différence significative n'a été observée sur les caractéristiques des diodes obtenues.

4.5 Qualité DU Clivage. - Sans tenter de faire une étude systématique de ce point, nous avons néanmoins constaté un léger effet de la qualité du clivage sur la hauteur de barrière et les densités des états $A$ et $\mathrm{B}$ : elles sont plus faibles si la diode est réalisée sur une zone mal clivée.

4.6 Présence D’états DE SURFACE. - Nous distinguerons ici deux points :

- la présence d'états de surface "naturels " ne semble pas jouer un rôle important. Le petit effet de la qualité du clivage est peut-être associé à la présence d'états dus aux imperfections de la surface clivée. De même, la comparaison des contacts Al-GaAs sur les surfaces $\langle 110\rangle$ et $\langle 100\rangle$ montre une barrière et une densité d'états $\mathrm{A}$ un peu plus élevées pour la face $\langle 110\rangle$. Cependant, la présence ou l'absence d'états de surface intrinsèques n'est pas le seul paramètre modifié dans ce cas et une conclusion nette est prématurée.

- par contre, si on crée des états « artificiels » en grande densité, par exemple par bombardement de la surface [13] la perturbation est très forte. La hauteur de barrière diminue considérablement (de plus de $0,3 \mathrm{eV}$ ) et la distribution des états est complètement modifiée.
4.7 INTERDIFFUSION. - Un recuit de $150 \mathrm{~min}$. à $200{ }^{\circ} \mathrm{C}$ d'un contact Au-GaAs clivé provoque des modifications de barrière et de distribution d'états qualitativement semblables à ce qui est observé après bombardement ionique.

4.8 Pollution De L'INTERfaCe. - Des essais de métallisation d'or et d'argent sur des surfaces clivées sous une faible pression partielle d'oxygène $\left(10^{-6}\right.$ torr $)$ ont donné des résultats contradictoires : il n'y a aucun effet dans le cas de l'argent (de même qu'un nettoyage chimique de la surface est pratiquement sans influence : cf. Fig. 10). Par contre, dans le cas de l'or, la barrière diminue et le facteur d'idéalité $n$ augmente pendant que la densité des états $\mathrm{A}$ et $\mathrm{B}$ diminue et que celle des états $\mathrm{C}$ augmente. L'adsorption d'oxygène est connue pour "désordonner » la surface du GaAs [14]; il semble que cet effet puisse être guéri par le dépôt de certains métaux $(\mathrm{Ag})$ et pas par d'autres (Au).

\section{Conclusion.}

Nous avons effectué des études comparatives des caractéristiques électriques et de la distribution des états d'interface pour des contacts Ag, Au et Al-GaAs. Ces contacts ont été préparés de façons diverses afin de modifier un grand nombre de paramètres physiques pouvant avoir une influence sur la hauteur de barrière.

Les principales conclusions de cette étude, sont :

- il existe trois types d'états d'interface dans la partie supérieure de la bande interdite du GaAs. Ces états sont situés au voisinage du niveau de Fermi du métal (états $\mathrm{A}$ ), vers $E_{\mathrm{c}}-0,4 \mathrm{eV}$ (états $\mathrm{B}$ ) et près $\mathrm{du}$ bas de la bande de conduction (états $\mathrm{C}$ );

- il existe une corrélation entre la densité des états $\mathrm{A}$ et la hauteur de barrière;

- la présence d'états de type $\mathbf{C}$ en grande densité est corrélée à l'apparition de non-idéalités sur les caractéristiques $I-V$ (excès de courant à faible polarisation, paramètre $n$ élevé);

- le travail de sortie du métal, la composition atomique superficielle, la qualité du clivage et l'orientation cristalline de GaAs, n'ont que peu - ou pas d'influence sur les paramètres de l'interface. La réactivité chimique du métal vis-à-vis de GaAs semble jouer un rôle un peu plus important;

- pour atténuer de façon significative la barrière de Schottky et la distribution des états d'interface, il faut créer, entre le métal et le semiconducteur, une zone interfaciale épaisse de plusieurs couches atomiques et fortement perturbée.

\section{Remerciements.}

Nous tenons à remercier tout particulièrement M. J. Massies (LPSES, Valbonne) pour la réalisation des contacts Al-GaAs par EJM au LCR ThomsonCSF et MM. J. M. Palau et A. Ismail (LESIC, Montpellier) pour la réalisation des contacts sur GaAs clivé et bombardé. 


\section{Bibliographie}

[1] Brillson, L. J., Surf. Sci. Rep. 2 (1982) 123.

[2] Bolmont, D., Chen, P., Sebenne, C. A., Surf. Sci. 117 (1982) 417.

[3] Williams, R. H., J. Vac. Sci. Technol. 18 (1981) 929.

[4] Spicer, W. E., Chye, P. W., Skeath, P. R., Su, C. Y., Lindau, I., J. Vac. Sci. Technol. 16 (1979) 1422.

[5] Bardeen, J., Phys. Rev. 71 (1947) 717.

[6] Barret, C., Vapaille, A., J. Appl. Phys. 50 (1979) 4217.

[7] Crowell, C. R., Beguwala, M., Solid State Electron. 14.(1971) 1149.

[8] Barret, C., Chekir, F., Vapaille, A., J. Phys. C 16 (1983) 2421.
[9] Massies, J., Linh, N. T., Surf. Sci. 114 (1982) 147.

[10] Michaelson, M. B., J. Appl. Phys. 48 (1977) 4728.

[11] Massies, J., Etienne, P., Dezaly, F., Linh, N. T., Surf. Sci. 99 (1980) 121.

[12] Barret, C., Chekir, F., Neffati, T., Vapaille, A., Massies, J., Proc. XVIth Int. Conf. Physics of Semiconductors, Montpellier 1982, Physica B 117118 (1983) 851.

[13] Palau, J. M., Ismail, A., Testemale, E., LassabaTERE, L., Proc. XVIth Int. Conf. Physics of Semiconductors, Montpellier 1982, Physica B 117-118 (1983) 860.

[14] Spicer, W. E., Chye, P. W., Garner, C. M., Lindau, I., Pianetta, P., Surf. Sci. 86 (1979) 763. 